\title{
Comparaison des diversités génétiques de Dacryodes edulis (G.Don) H.J. Lam et de Dacryodes buettneri (Engel.) H.J. Lam (Burséracées), deux espèces forestières utiles en Afrique centrale
}

\author{
Gilbert TODOU ${ }^{1,2,4^{*}}$, Laure BENOIT ${ }^{2}$, Geo COPPENS d'EECKENBRUGGE ${ }^{2}$, \\ Hélène I. JOLY ${ }^{2}$, Jean-Michel ONANA ${ }^{3}$, ACHOUNDONG $^{3}$ et AMOUGOU AKOA ${ }^{4}$ \\ ${ }^{1}$ Université de Maroua, École Normale Supérieure, BP : 55, Maroua, Cameroun. \\ ${ }^{2}$ CIRAD-Bios/UMR 5175, CEFE, 1919 Route de Mende 34293 Montpellier Cedex 5, France. \\ ${ }^{3}$ IRAD, Herbier National du Cameroun, BP : 1601, Yaoundé, Cameroun. \\ ${ }^{4}$ Université de Yaoundé I, Faculté des Sciences, BP : 812, Yaoundé, Cameroun. \\ *Auteur correspondant, E-mail : gitodou@ hotmail.com; Tél. : +23796964151
}

\section{RESUME}

Dacryodes buettneri et Dacryodes edulis sont des espèces à usages multiples en Afrique centrale. Elles servent principalement de bois d'œuvre et de fruitier. Des échantillons de leurs folioles ont été récoltés sur 694 arbres répartis dans 29 populations au Cameroun et au Gabon, afin d'extraire l'ADNn et amplifier cinq marqueurs microsatellites. Les paramètres génétiques des populations ont été calculés pour évaluer et comparer leur diversité génétique. Les moyennes des nombres d'allèles sont respectivement $4,43 \pm 1,37$ et 5,36 $\pm 1,13$. Il n'existe pas de différence significative dans la répartition des nombres d'allèles par locus entre les espèces. Les paramètres de la diversité intra-population de D. buettneri $\left(\mathrm{P}=0,76 \pm 0,15 ; \mathrm{H}_{\text {att }}=0,35 \pm 0,06\right.$ et $\mathrm{H}_{\mathrm{obs}}=0,26 \pm$ $0,04)$ sont significativement inférieurs à ceux de D. edulis $\left(\mathrm{P}=0,9 \pm 0,12 ; \mathrm{H}_{\mathrm{att}}=0,47 \pm 0,05\right.$ et $\mathrm{H}_{\mathrm{obs}}=0,41 \pm$ $0,07)$, alors que le coefficient de consanguinité de $D$. buettneri $(\mathrm{F}=0,25 \pm 0,08)$ est deux fois supérieur à celui de la deuxième espèce $(\mathrm{F}=0,12 \pm 0,03)$. Un important flux de gènes inter-espèce a été trouvé, $\mathrm{Nm}=0,44$. Les analyses ont ressorti trois groupes de populations, séparés par la distance géographique et l'isolement taxonomique. $D$. edulis se croise parfaitement si les individus sont géographiquement proches, même avec les individus spontanés forestiers. Aucune séparation variétale au sein de D. edulis n'a été détectée. Cette étude est une contribution à la valorisation de la diversité génétique des espèces forestières africaines pour une conservation durable.

(C) 2013 International Formulae Group. All rights reserved.

Mots clés : Espèces à usages multiples, marqueurs microsatellites, génétique de populations, conservation durable.

\section{INTRODUCTION}

Dacryodes edulis (G.Don) H.J. Lam (noms locaux: safoutier ou prunier) et Dacryodes buettneri (Engel.) H.J. Lam (nom commercial : ozigo) appartiennent à la famille des Burséracées, toutes originaires des forêts humides du golfe de Guinée (Verheij, 2002; Onana, 2008). D. buettneri n'est pas cultivé. Il crô̂t en milieu naturel localement dense, centré sur le Gabon. Alors que D. edulis est une espèce semi-domestiquée qui est diffusée hors de sa distribution originelle, dans une 
vingtaine de pays (Verheij, 2002). A l'état naturel, ces deux espèces sont de la canopée supérieure mais les safoutiers en plantation excèdent rarement $12 \mathrm{~m}$ de hauteur.

Ces espèces sont dites à usages multiples. D. edulis, espèce cultivée, est un fruitier dont les fruits sont très appréciés par les populations. Riches en matières nutritives pour l'Homme (Mbofung et al., 2002), ils sont mangés après cuisson ou braisage. Ils font l'objet des transactions commerciales, même au-delà des frontières africaines (Awono et al., 2002; Tabuna, 1999; 2000). D. buettneri, espèce non cultivée, est comptée parmi les cinq premières essences à bois d'œuvre exploitées au Gabon, et alimente les marchés nationaux et internationaux (Todou et Doumenge, 2008). Au Gabon, D. buettneri est un important arbre fruitier. Ses fruits ressemblent à ceux du safoutier $(D$. edulis (G.Don) H.J.Lam), mais ils sont plus petits et ne sont pas vendus. En plus des usages principaux, des différentes parties de ces espèces sont utilisées dans la pharmacopée traditionnelle (Ajibesin, 2011) et les petites branches servent de bois de chauffage.

Les deux espèces sont proches morphologiquement, mais le safoutier se distingue par deux petites folioles cordiformes et acuminées, très rapprochées de la base du pétiole (Figure 1). Alors que l'ozigo se caractérise par des folioles poilues, de couleur brun rougeâtre en-dessous. Son écorce externe du tronc est jaune dorée et s'écaille en longues et larges lames (Figure 2). Génétiquement, les deux espèces semblent proches car cinq marqueurs microsatellites ont été isolés de $D$. edulis et ont pu être amplifiés avec succès sur $D$. buettneri (Benoit et al., 2011).

Le but de cette étude est de contribuer à la valorisation de la diversité génétique et de conserver durablement les ressources phytogénétiques des espèces forestières africaines. Les objectifs spécifiques consistent à calculer et comparer les paramètres génétiques des populations des deux espèces, à évaluer le flux de gènes entre les espèces et à déterminer la structure génétique au sein des espèces.
MATERIEL ET METHODES

Récolte du matériel végétal et échantillonnage des populations

Le matériel végétal, folioles de $D$. buettneri et $D$. edulis, a été récolté pour extraire de l'ADNn de 2006 à 2010 au Cameroun et au Gabon. Les folioles récoltées ont été séchées au gel de silice et conservées dans des sacs en plastique hermétiquement fermés. Tout le matériel collecté (avec échantillons d'herbiers à l'appui) a été identifié en utilisant la clé de détermination développée par Lam (1932) considérant les révisions nommenclaturales de Pierlot (1996); Onana $(1998,2008)$ et Onana et Cheek (2003).

Les arbres prospectés ont été groupés en populations selon leur rapprochement géographique. L'étude a été basée sur un échantillonnage de 29 populations dont 18 pour D. edulis au Cameroun, 5 pour D. edulis au Gabon et 6 populations de D. buettneri au Gabon (Figure 3). Les folioles de D. buettneri (170 au total) ont été récoltées uniquement en milieu forestier naturel. Celles de D. edulis du Cameroun (400 au total) ont été récoltées dans les plantations (14 populations), dans les vieilles habitations ou des endroits plus ou moins anthropisés (RF Mokoko, Rhumpi et NNO Manfé) et dans les milieux naturels forestiers non perturbés (Bibondi-F). Pour $D$. edulis du Gabon (124 au total), les folioles ont été récoltées uniquement dans les plantations. Au total, 694 folioles (de 694 arbres) ont été récoltées. Les échantillons récoltés sont conservés au laboratoire du Service des Marqueurs Génétiques du CEFE à Montpellier (France) et les échantillons d'herbiers sont conservés à l'Herbier National du Cameroun (YA) et au Musée d'Histoire Naturelle (P) à Paris (France).

\section{Extraction d'ADN et conditions d'amplification des microsatellites}

Les fragments de $10 \mathrm{mg}$ de folioles séchées ont été finement broyés avec une bille de tungstène par agitation dans un broyeur Retsch MM301. Les ADNn ont été extraits suivant le protocole de DNeasy 96 Plant Kit (Qiagen, Hilden, Germany 2006). 
L'amplification des ADNn a été faite à partir de cinq marqueurs microsatellites isolés de D. edulis (Benoit et al., 2011) dont les caractéristiques sont présentées dans le Tableau 1. Le multiplexe PCR Kit (Qiagen, Hilden, Germany 2006) a été fait en suivant le protocole fourni pour un volume de $10 \mu \mathrm{L}: 5$ $\mu \mathrm{L}$ de $2 \times$ Qiagen multiplexe Master Mix, 1 $\mu \mathrm{L}$ d'amorces mix (mix d'amorces complémentaires; $2 \mu \mathrm{M}$ ), $1 \mu \mathrm{L}$ de $\mathrm{H}_{2} 0$ et $2 \mu \mathrm{L}$ de l'ADN extrait. Les amplifications de l'ADNn ont été réalisées dans les thermocycleurs (eppendorf Mastercycler gradient) au laboratoire de Service Commun des Marqueurs Génétiques du CEFE Montpellier.

\section{Séquençage des ADN extraits}

Le produit PCR a été purifié sur colonne (QiAquick 96 PCR purification kit, Qiagen, Hilden, Germany 2006). La réaction de séquençage a été réalisée dans les sens direct et inverse en utilisant le kit Big Dye 3.1 chemistry (Applied Biosystems, Lennik, PaysBas). Un microlitre du produit PCR dilué (1/100) a été ajouté à $15 \mu \mathrm{l}$ de formamide désionisé et $0,2 \mu \mathrm{l}$ de LIZ (marqueur de taille marqué par un fluorochrome). Le génotypage a été fait sur le séquenceur 16 capillaires ABI PRISM par l'IFR 119 de l'Université Montpellier 2, France.

Calculs et analyses des paramètres de génétique de populations

Les résultats de l'amplification d'ADNn recouvrés électroniquement par le séquenceur ont été traités sous le logiciel GENEMAPPER 3.5 software (Applied Biosystems, Foster City, California, USA). Ce logiciel a déterminé le nombre d'allèles de chaque locus et a assigné une taille (en nombre de paires de bases) à chaque allèle. Six paramètres de génétique des populations ont été calculés sous GENETIX 4.05 (Belkhir et al., 2004) différemment pour les deux espèces. Ils ont été comparés et interprétés. Ce travail a utilisé la loi de Hardy-Weinberg pour tester si les populations sont en équilibre panmictique. Le logiciel STRUCTURE 2.3.3 a été utilisé pour déterminer la structure génétique et les différents groupes génétiques homogènes qu'on pourrait trouver au sein des espèces.

- Le nombre moyen d'allèles par locus (A) est le rapport de la somme totale des allèles sur le nombre de locus étudiés. A a été calculé de la manière suivante :

$A=\frac{\sum_{i=1}^{n} a t}{L}$

où ai est le nombre d'allèles au locus $\mathrm{i}$ et $L$ est le nombre des locus étudiés.

- Le taux de polymorphisme (P) est le nombre de locus polymorphes par rapport au nombre de locus étudiés. $\mathrm{P}$ a été calculé de la manière suivante :

$$
P=\frac{x}{m}
$$

où $x$ est le nombre de locus polymorphes et $m$ est le nombre total de locus.

Le taux de polymorphisme moyen pour une espèce est la moyenne des taux de polymorphisme des différentes populations étudiées.

L'hétérozygotie exprime la probabilité pour que deux gènes tirés au hasard dans une population soient différents. Elle est définie par $H_{\text {att }}$ (hétérozygotie attendue), équivalent à l'hétérozygotie théorique sous l'hypothèse de HardyWeinberg. Dans une population en équilibre panmictique, chaque génotype a pour fréquence, le produit de fréquence des allèles (pi) qui le constituent.

Le taux d'hétérozygotie attendue $\left(H_{a t t}\right)$ au locus $k$ a été calculé de la manière suivante :

$H_{a t t}=1-\sum_{i=1}^{a k} p^{2} i k$

$a k$ est le nombre d'allèles au locus $k$.

Le taux d'hétérozygotie observée $\left(H_{o b s}\right)$ au locus $\mathrm{k}$ est calculé de la manière suivante :

$H_{o b s k}=\sum_{i j=1}^{a k} p t k(i \neq j)$

pij est l'estimation de la fréquence du génotype $i j$ au locus $k$. 

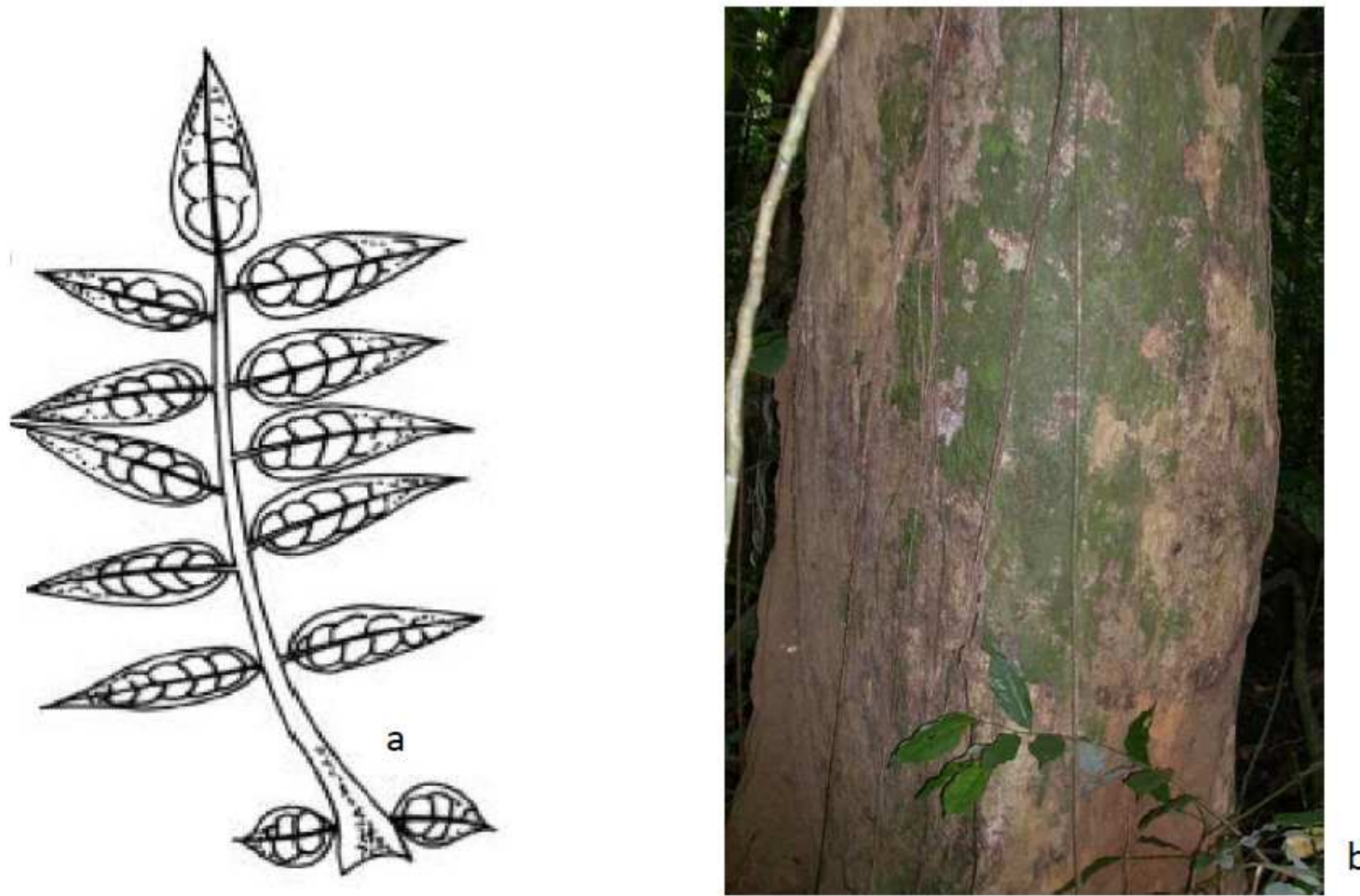

Figure 1 : Détails morphologiques (a) des feuilles et (b) du tronc de D. edulis.

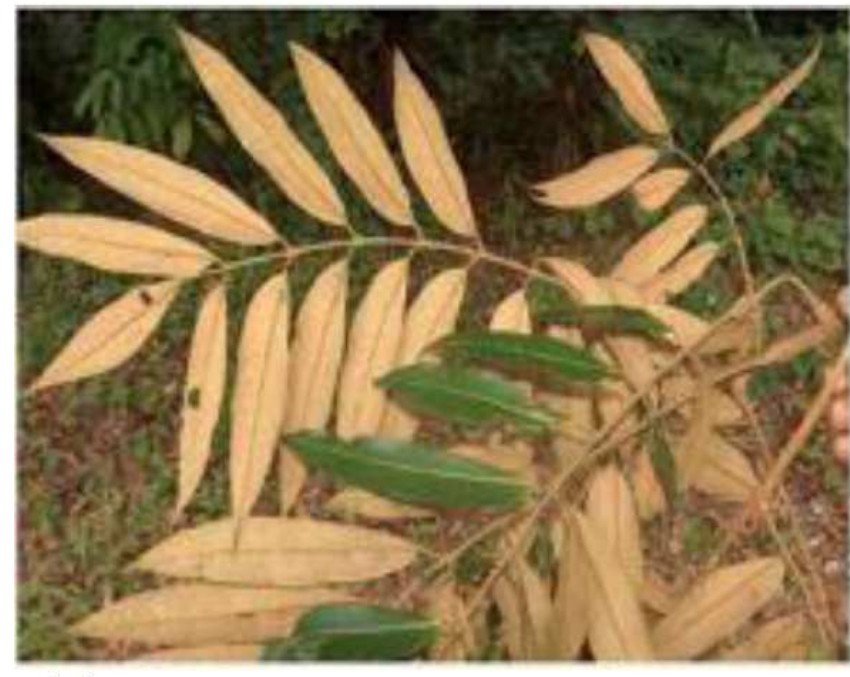

(a)

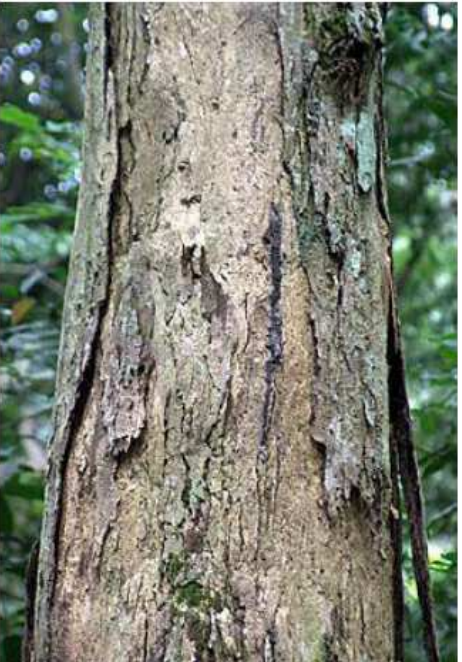

(b)

Figure 2 : Détails morphologiques (a) des feuilles et (b) du tronc de D. buettneri. 


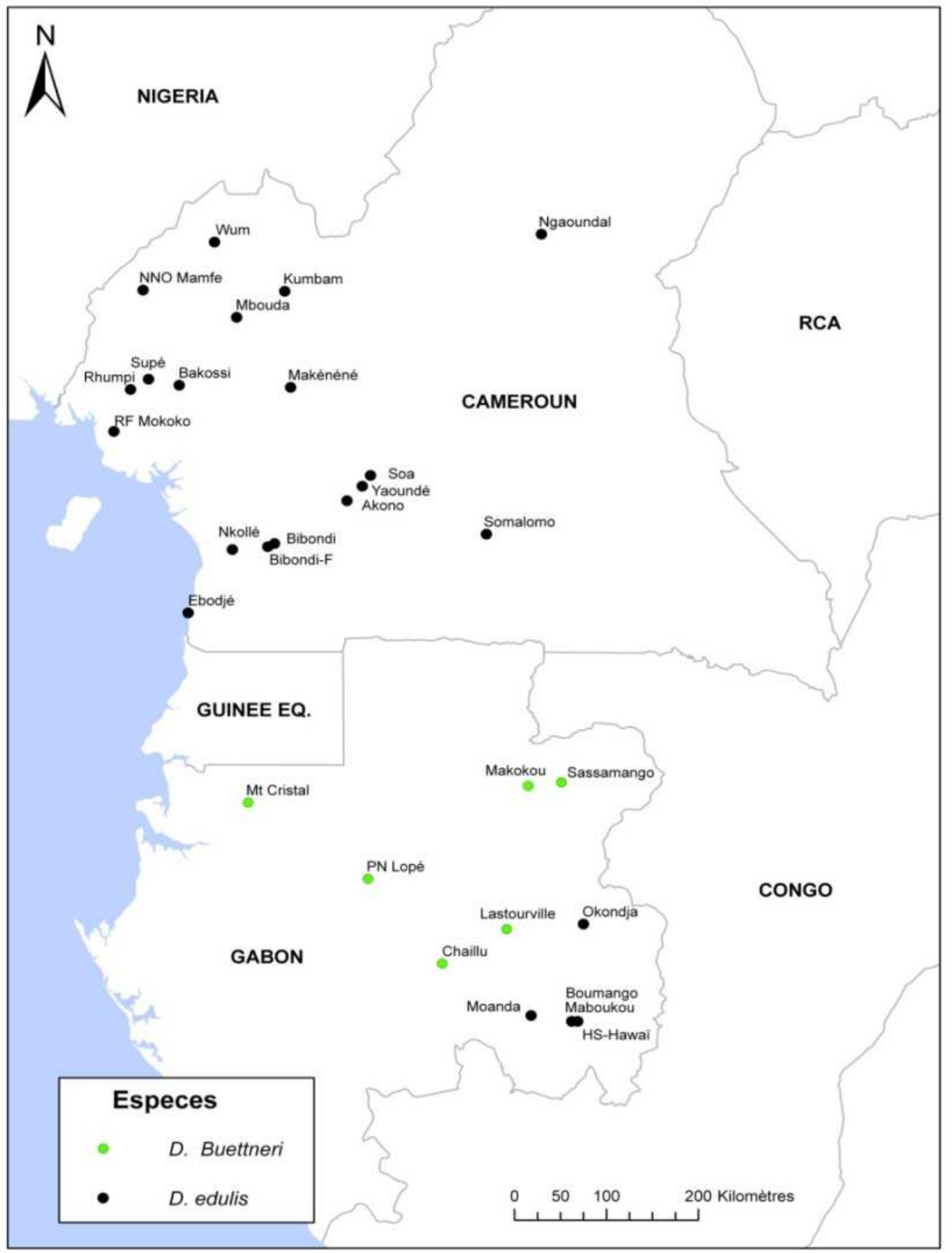

Figure 3 : Distribution des populations échantillonnées étudiées.

Tableau 1 : Caractéristiques des marqueurs microsatellites utilisés pour étudier la diversité génétique de $D$. buettneri et $D$. edulis. 


\begin{tabular}{lcl}
\hline Noms des marqueurs & Motifs de bases & Séquences des amorces $\left(\mathbf{5}^{\prime}>\mathbf{3}\right.$ ') \\
\hline CB09 & (GA) 10 & F: CAGTGGTAAAGGTAGGATGG \\
& & R: GGTTTGTGTTGGTTGTTTTC \\
\hline LD06 & (GA) 10 & $\begin{array}{l}\text { F: CATGTGCATCTTTTGATCTC } \\
\text { R: TAGAGGGGAAAGGTAGAAGG }\end{array}$ \\
& & F: CACACGATCAAGAAAGACC \\
\hline CG11 & (CT) 8 & F: CCGTAATGACTCTCTAATTG \\
\hline LB12 & (GA) 14 & F: AGACCACAAGAACCTTTGC \\
& & F: GGCACTTAATTTGATTTTG \\
\hline CE09 & (GA) 16 & R: GTAGCTGGGAGGAGATCAGG \\
& & R: ACAGCCCCAAAAGCAC \\
\hline
\end{tabular}

Les taux d'hétérozygotie attendue $\left(H_{a t t}\right)$ et d'hétérozygotie observée $\left(H_{o b s}\right)$ d'une espèce représentent la moyenne arithmétique d'hétérozygotie correspondant à des différents locus étudiés.

- L'écart à la panmixie est l'indice de fixation ou encore coefficient de consanguinité $(F)$. Il traduit la mesure de l'écart à la structure de Hardy-Weinberg. Il varie entre -1 et +1 et permet de connaitre le déficit ou l'excès en hétérozygote dans une population. Il est calculé de la manière suivante :

$$
F=1-\frac{H_{o b s}}{H_{a t t}}
$$

$\mathrm{Si} F$ est négatif, il y a excès en hétérozygote dans la population étudiée et si $F$ est positif, il y a déficit en hétérozygote dans la population étudiée.

- Le coefficient de la différenciation génétique entre les populations est noté $F$ st. Il exprime le degré de la similarité génétique ou de la dissemblance génétique de deux populations. La valeur de $F s t$ a été calculée de la manière suivante :

$$
F_{\mathrm{ST}}=1-\frac{\left(1-\mathrm{F}_{\mathrm{IT}}\right.}{\left(1-\mathrm{F}_{\mathrm{IS}}\right.}
$$

F-statistiques (Fst, Fit et Fis) expriment l'organisation génétique dans une population totale divisée en sous-populations (Weir et Cockerham, 1984). Ils ont été obtenus par le programme de logiciel Genetix. Selon Wright (1978), si $0<F s t<0,05$, la différenciation génétique est considérée comme faible; si 0,05 $<F s t<0,15$, la différenciation génétique est considérée comme modérée; si $0,15<F s t<$ 0,25 alors la différenciation génétique est considérée comme importante et si $F s t>0,25$ alors la différenciation génétique est considérée comme très importante.

- Le flux de gènes $(\mathrm{Nm})$ estime le degré d'échange de gènes entre les populations. La différenciation génétique entre populations est favorisée par la dérive et limitée par les flux de gènes entre populations. Le taux de migration $\mathrm{m}$ est relié à la différenciation génétique Fst par la relation suivante :

$$
N m=\frac{1-F s t}{4 * F s t}
$$

$N$ représente le nombre effectif d'individus d'une population.

\section{RESULTATS}

Diversité génétique intra-population de Dacryodes buettneri et Dacryodes edulis

Le nombre d'allèles moyens par locus chez $D$. buettneri varie de $2,33 \pm 1,50$ pour LD09 à 7,83 $\pm 1,83$ pour CB09 avec une moyenne totale de $4,43 \pm 0,93$. Il varie de 2,34 $\pm 0,98$ pour CG11 à $9,52 \pm 4,25$ pour CE0 9 chez $D$. edulis avec une moyenne totale de $5,40 \pm 1,13$. Les tailles d'allèles par locus sont différentes selon les espèces (Tableau 2). Bien 
que les nombres d'allèles par locus chez $D$. buettneri soient légèrement inférieurs à ceux de $D$. edulis, il n'y a pas de différence statistiquement significative entre les espèces. Les locus les plus polymorphes sont CB09 et CE09 pour les deux espèces. Les autres paramètres de la diversité génétique intrapopulation de $D$. buettneri $(\mathrm{P}=0,76 \pm 0,15$; $H_{a t t}=0,35 \pm 0,06$ et $\left.\mathrm{H}_{\mathrm{obs}}=0,26 \pm 0,04\right)$ sont significativement inférieurs à ceux de $D$. edulis (Tableau 3). Le coefficient de consanguinité de D. buettneri $(\mathrm{F}=0,25 \pm$ $0,08)$, une espèce exploitée à l'état sauvage, est deux fois supérieur à celui de D. edulis (F $=0,12 \pm 0,03)$, une espèce semi-cultivée.

\section{Diversité génétique inter-population de Dacryodes buettneri et Dacryodes edulis}

La différenciation génétique moyenne entre les populations de $D$. edulis $(F s t=0,03)$ est faible par rapport à celle de $D$. buettneri $(F s t=0,08)$. Elle indique que la grande partie de la diversité génétique des espèces est d'origine intra-population, seulement $3 \%$ et $8 \%$ de diversité sont d'origine interpopulation. La valeur de flux de gènes de $D$. edulis $(N m=6)$ est plus du double de celle de D. buettneri $(\mathrm{Nm}=2,87)$ (Tableau 3$)$.
Rapprochement génétique interspécifique, Dacryodes buettneri - Dacryodes edulis

Le flux de gènes entre $D$. buettneri et D. edulis est de $N m=0,44$ (Tableau 4) indiquant que moins d'un migrant passe d'une espèce à une autre au cours de deux générations. Cette valeur est importante pour deux espèces différentes. La distance génétique moyenne entre les deux espèces est Fst $=0,74$. Cette valeur est considérée très importante.

L'analyse factorielle de correspondances, AFC (Figure 4) basée sur le centre de gravité des populations a ressorti trois groupements de populations (groupe $\mathrm{I}=$ groupe de D. edulis du Cameroun; groupe II = groupe de D. edulis du Gabon et groupe III = groupe de $D$. buettneri). Aucun autre groupe d'individus n'est détecté. Les individus de $D$. edulis semblent plus dispersés sur le plan F1F3 que ceux de $D$. buettneri, justifiant ainsi une différence de niveau de diversité génétique entre les espèces. Les populations de $D$. edulis sont génétiquement plus diversifiées que celles de $D$. buettneri. L'analyse de l'histogramme de la structure génétique (Figure 5) a ressorti les trois groupes de populations révélés par l'analyse factorielle de correspondances, séparés par la distance géographique et par l'isolement taxonomique.

Tableau 2 : Nombres moyens d'allèles et tailles d'allèles locus.

\begin{tabular}{ccccc}
\hline Loci & \multicolumn{2}{c}{ D. buettneri } & \multicolumn{2}{c}{ D. edulis } \\
\cline { 2 - 5 } & A & PB & A & PB \\
\hline CB09 & $7,83 \pm 1,83 \mathrm{a}$ & $167-192$ & $5,65 \pm 1,84 \mathrm{a}$ & $163-200$ \\
CE09 & $7,33 \pm 3,14 \mathrm{a}$ & $126-159$ & $9,52 \pm 4,25 \mathrm{a}$ & $152-175$ \\
CG11 & $2 \pm 0,63 \mathrm{~b}$ & $175-183$ & $2,34 \pm 0,98 \mathrm{~b}$ & $170-185$ \\
LB12 & $2,66 \pm 1,21 \mathrm{~b}$ & $210-230$ & $3,70 \pm 1,45 \mathrm{~b}$ & $205-230$ \\
LD09 & $2,33 \pm 1,50 \mathrm{~b}$ & $146-164$ & $5,74 \pm 4,43 \mathrm{ab}$ & $125-168$ \\
Moyennes & $4.43 \pm 0.93$ & - & $5,40 \pm 1,13$ & - \\
\hline
\end{tabular}

$\mathrm{A}=$ nombre d'allèles par locus; $\mathrm{PB}=$ taille d'allèles en paire de bases. 
Tableau 3 : Paramètres de génétique de populations de Dacryodes buettneri et de Dacryodes edulis.

\begin{tabular}{lcccccc}
\hline & \multicolumn{5}{c}{ Paramètres génétiques } \\
\cline { 2 - 6 } Espèces & $\mathbf{P}$ & $\mathbf{H}_{\mathrm{att}}$ & $\mathbf{H}_{\text {obs }}$ & $\mathbf{F}$ & $\mathbf{F s t}$ & $\mathbf{N m}$ \\
\hline D. buettneri & $0,76 \pm 0,15 \mathrm{a}$ & $0,35 \pm 0,06 \mathrm{~b}$ & $0,26 \pm 0,04 \mathrm{~d}$ & $0,25 \pm 0,08 \mathrm{f}$ & $0,08 \mathrm{~h}$ & $2,87 \mathrm{j}$ \\
D. edulis & $0,9 \pm 0,12 \mathrm{a}$ & $0,47 \pm 0,05 \mathrm{c}$ & $0,41 \pm 0,07 \mathrm{e}$ & $0,12 \pm 0,03 \mathrm{~g}$ & $0,03 \mathrm{i}$ & $6,00 \mathrm{k}$ \\
\hline \multicolumn{2}{c}{$P=$ pourcentage de polymorphisme; $H_{\text {obs }}=$ hétérozygotie observée; $H a_{t t}=$ hétérozygotie attendue; $F=$ coefficient de } \\
consanguinité; $F$ st $=$ coefficient de différenciation génétique; $N m=$ flux de gènes.
\end{tabular}

Tableau 4 : Matrice des moyennes de flux génétique (Nm) (au-dessus de la diagonale) et de la différenciation génétique (Fst) (en dessous de la diagonale) entre D. buettneri et D. edulis.

\begin{tabular}{llc}
\hline \multirow{2}{*}{ Espèces } & \multicolumn{2}{c}{ Espèces } \\
\cline { 2 - 3 } D. edulis & D. edulis & D. buettneri \\
D. buettneri & - & 0,44 \\
\hline
\end{tabular}

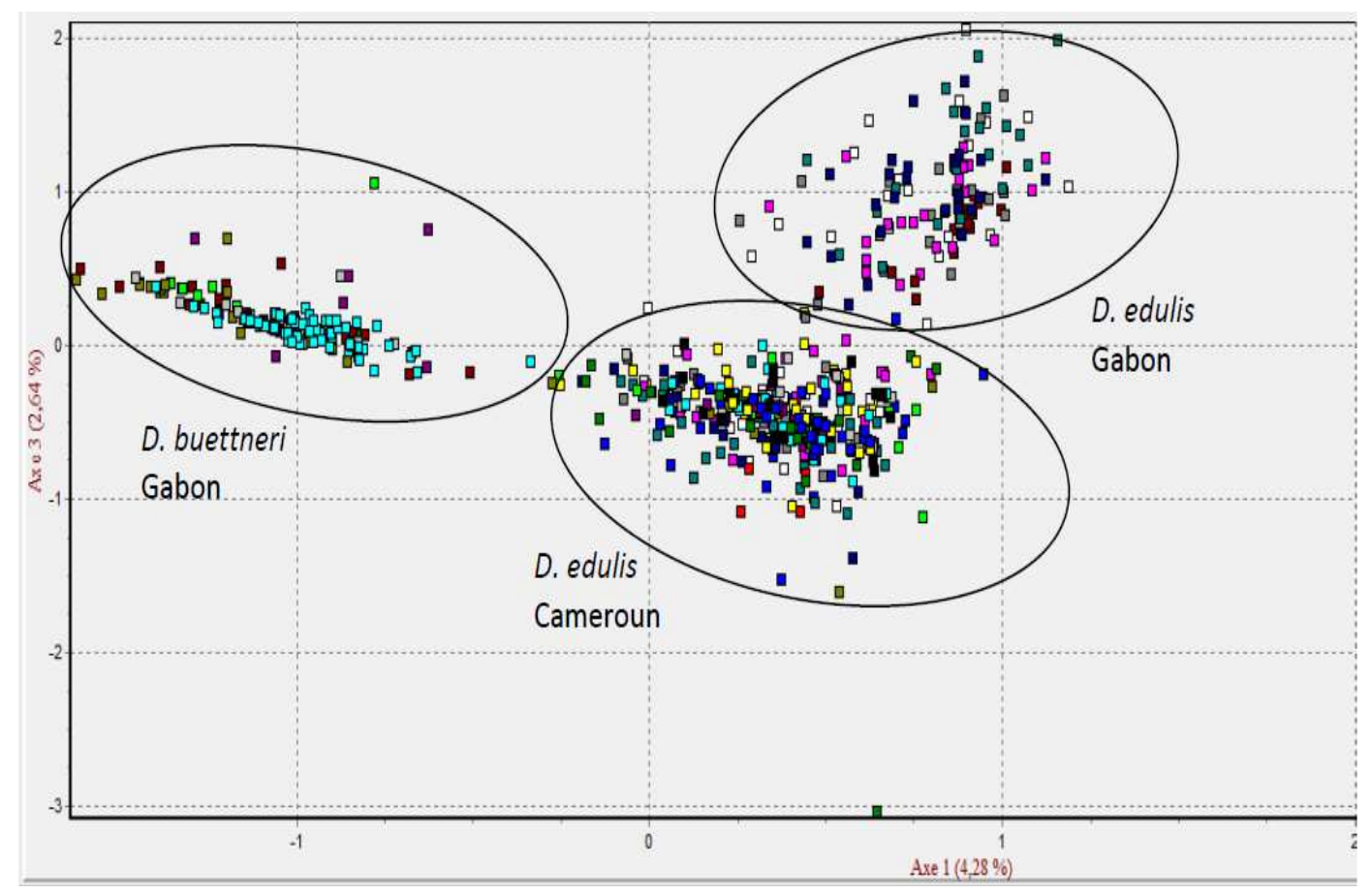

Figure 4 : Projection sur le plan 1-3 de l'AFC des individus des populations de $D$. edulis et de $D$. buettneri. 


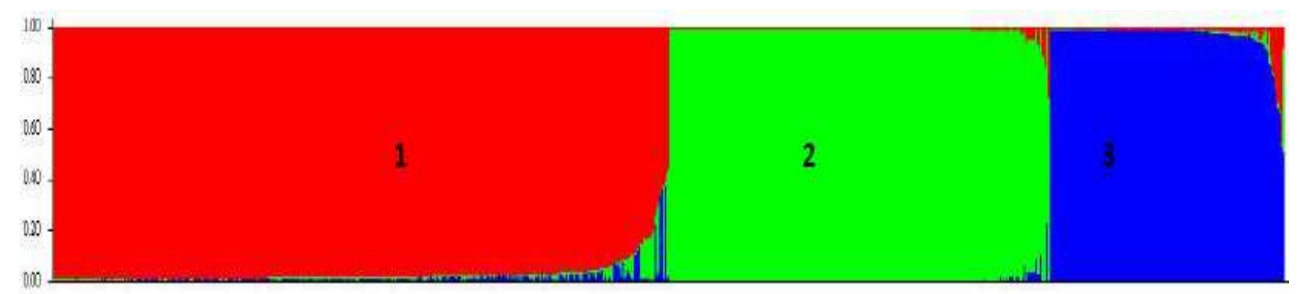

Figure 5 : Histogramme de la structure génétique de 694 individus au sein de $D$. edulis et de $D$. buettneri en Afrique centrale. Les plages de couleurs sont constituées de rectangles verticaux et chaque rectangle représente un individu. Chaque couleur représente un groupe génétique. En abscisse: 1- groupe génétique D. edulis récolté au Cameroun (400 individus); 2- groupe génétique $D$. edulis récolté au Gabon (124 individus) et 3- groupe génétique $D$. buettneri récolté au Gabon (90 individus). En ordonnée, la proportion d'affinité à un réservoir génétique c'est-à-dire la proportion de chaque réservoir génétique dans un individu.

\section{DISCUSSION}

Niveau de diversité génétique en accord avec les actions anthropiques et le système de reproduction des espèces

Les valeurs des hétérozygoties des deux espèces, D. buettneri $(\mathrm{A}=4,43 \pm 0.93$ et $\left.\mathrm{H}_{\mathrm{att}}=0,35 \pm 0,06\right)$ et celles de D. edulis $(\mathrm{A}=$ $5,40 \pm 1,13$ et $\left.\mathrm{H}_{\text {att }}=0,47 \pm 0,05\right)$ sont inférieures à celles générées pour Entandrophragma cylindricum dont $\mathrm{A}=$ 18,5 et $\mathrm{H}_{\text {att }}=0,85$ (Garcia et al., 2004). Vignes et al. (2006) ont trouvé une hétérozygotie attendue $\mathrm{H}_{\text {att }}=0,349$ pour Ficus insipida alors que Born et al. (2006) travaillant sur Aucoumea klaineana ont trouvé $\mathrm{A}=5,2$ et $\mathrm{H}_{\mathrm{att}}=0,46$. Bien que la diversité génétique de $D$. buettneri soit légèrement inférieure à celle de $D$. edulis, elle paraît plus élevée par rapport à la plupart des espèces tropicales soudaniennes qui ont été étudiées au moyen de l'électrophorèse enzymatique, à l'exemple de Vitellaria paradoxa dont $\mathrm{H}_{\mathrm{att}}=$ 0,214 (Lovett et Haq, 2000). D. buettneri présente moins d'hétérozygote que $D$. edulis car la valeur de son coefficient de consanguinité $(F=0,25 \pm 0,08)$ est deux fois supérieure à celle de la deuxième espèce $(\mathrm{F}=$ $0,12 \pm 0,03)$.

La valeur de ce coefficient relativement élevée est en adéquation avec la consanguinité observée au sein des espèces forestières en générale. En effet, D. buettneri et $D$. edulis sont des espèces dioïques (Onana, 2008; Todou et Doumenge, 2008) mais il existe des individus qui portent à la fois des fleurs mâles et des fleurs femelles, et des individus qui portent des fleurs hermaphrodites. Cette situation combinée avec la répartition naturelle de $D$. buettneri favorise l'autogamie qui entraîne un déficit important en hétérozygote. En plantation, les individus de D. edulis résultent d'apports génétiques hétérogènes et de sélections dans les milieux écologiques différents et dans les circonstances ethnologiques différentes (usages locaux, goûts, systèmes de production). La création d'une plantation de safoutiers au moyen des semences provenant d'endroits différents est une explication à la grande diversité génétique et l'excès en hétérozygote dans les populations. La densité relativement importante des arbres en champs (Kengue, 2002) combinée à une floraison synchronisée des arbres et le pouvoir pollinisant des insectes (Kengue et al., 2002) favoriseraient un brassage des gènes au sein des populations. Elles constituent des conditions favorables au maintien d'un niveau élevé de la diversité génétique, réduisant le taux d'homozygotes et augmentant le pourcentage de polymorphisme. Les safoutiers sont utilisés en champs comme ombrage aux caféiers et cacaoyers. Toutefois, des vergers purs de safoutiers ont été observés au Cameroun. Il est normal que $D$. edulis, une espèce forestière africaine cultivée en plantation, présente un indice de fixation $(F=$ 0,12 ) équivalent à la moitié de celui de $D$. 
buettneri $(F=0,25)$, une espèce sœur, mais exploitée à l'état sauvage. Les actions anthropiques ont aussi contribué à ce que $D$. edulis soit plus diversifié dans les populations que $D$. buettneri.

Il a été démontré que plus les populations sont géographiquement éloignées entre elles, plus elles auront tendance à être génétiquement différenciées, conséquence d'une baisse du flux de gènes (Loveless, 1992). Ce qui justifie les plus grandes valeurs de distances génétiques entre les populations du Cameroun et celles du Gabon. L'Homme a contribué à rapprocher génétiquement les individus de D. edulis par le processus de domestication étendant son aire de répartition. Par ailleurs, la création d'un champ de safoutiers se fonde sur des plantules ou des graines venant de plusieurs régions (BellaManga, 2000), rapprochant génétiquement les populations entre elles. La deuxième explication pourrait être le mode de dispersion des graines. L'Homme est le disperseur par excellence de D. edulis. Des plantules des safoutiers ont été observées dans les campements des chasseurs et des pisteurs des sociétés d'exploitation forestière en pleine forêts denses. De plus, les fruits et les plantules s'échangent entre les voisins, les communautés et/ou les villages voisins (Schreckenberg et al., 2002) facilitant la dispersion des graines. Les transactions commerciales permettent le transport des fruits, des graines, à une longue distance, d'autant plus que certains agriculteurs sélectionnent les fruits de bonne qualité sur les marchés pour créer leurs plantations.

\section{Aucun sous-groupe génétique détecté au sein des espèces}

Toutes les approches utilisées (AFC et histogramme de la structure génétique) ont montré l'appartenance des deux espèces à des groupes génétiques différents et aucun sousgroupe génétique n'a été détecté au sein des espèces. L'analyse génétique ne donne pas des délimitations tranchées justifiant une séparation variétale au sein des espèces.
Il semble que $D$. edulis se croise parfaitement si les individus sont géographiquement proches, même avec les individus des milieux forestiers naturels. Cette observation est en adéquation avec la dioécie de l'espèce. La pression de sélection sur cette espèce et les méthodes culturales sont encore artisanales pour influencer et créer des groupes génétiques de culture isolés. De surcroît, il existe dans cette aire des formes sauvages constituant une importante source de variabilité. Le flux génétique permanent entre les différentes composantes permet de maintenir la variation génétique, mais aussi de limiter la création de barrière génétique entre elles. Or, Okafor (1982) avait trouvé deux variétés au sein de l'espèce, délimitation basée sur les dimensions des fruits et l'architecture de l'arbre. L'analyse de la structure génétique a montré que ces caractères ne se reflètent pas au niveau génétique. Cette analyse justifie l'assertion d'Onana (2008) qui estimait que la clé utilisée pour distinguer une nouvelle variété au sein de $D$. edulis serait basée sur les caractères horticulturaux.

\section{Conclusion}

La répartition des nombres moyens d'allèles aux locus est similaire pour les espèces étudiées. Il n'y a pas de différence statistiquement significative entre les espèces. Cependant, $D$. edulis est génétiquement plus diversifié que $D$. buettneri et la différenciation génétique $(F s t)$ entre les populations est plus faible que la deuxième espèce. Le taux de consanguinité est plus élevé au sein de $D$. buettneri, espèce purement sauvage que $D$. edulis, espèce semi-domestiquée. Cette situation est due en partie aux actions anthropiques sur l'espèce par création des plantations. Comme pour la plupart des espèces forestières, la grande partie de la diversité génétique des espèces étudiées est d'origine intra-population. Cette étude a permis de noter un flux de gènes interspécifique non négligeable. Trois groupements de populations ont été notés, séparés par la distance géographique et par l'isolement taxonomique : groupe $\mathrm{I}=$ groupe 
de $D$. edulis du Cameroun; groupe II = groupe de $D$. edulis du Gabon et groupe III = groupe de D. buettneri. Mais, aucune délimitation tranchée justifiant une séparation variétale au sein de $D$. edulis n'a été prouvée par les analyses génétiques, ni une séparation entre les safoutiers spontanés en forêt et ceux cultivés en plantation.

Cette étude est une contribution à la valorisation de la diversité génétique des ressources phytogénétiques afin d'optimiser la conservation efficiente et l'utilisation durable des espèces forestières africaines.

\section{REMERCIEMENTS}

Les travaux de terrain et de laboratoire ont été menés en partie dans le cadre du projet IFORA (Îlots forestiers africains) financé par l'Agence Nationale de Recherche. Le personnel de l'Herbier national du Cameroun à Yaoundé a facilité des descentes de terrain, l'identifié et la confirmé des noms scientifiques des espèces. Sont remerciées toutes les personnes qui ont contribué à la réalisation de cette étude : Nana Victor, Charles Doumenge, Marie Gabeloux pour leur participation à la collecte du matériel végétal.

\section{REFERENCES}

Ajibesin KK. 2011. Dacryodes edulis (G. Don) HJ. lam. A review on its Medecinal, Phytochimical and Economical Properties. Research Journal of Medecinal Plant, 5: 32-41.

Awono A, Ndoye O, Schreckenberg K, Tabuna H, Isseri F, Temple L. 2002. Production and marketing of safou (Dacryodes edulis) in Cameroon and internationally: Market development issues. Forests, Trees and Livelihoods, 12: $125-147$.

Bella-Manga. 2000. Domestication des espèces sauvages et problèmes phytosanitaires. In Actes de 3è Séminaire international sur la valorisation du Safoutier et autres produits oléagineux non-conventionnels Kengue J, Kapseu C et Kayem GJ (eds). Yaoundé, Cameroun, 37-39.
Belkhir K, Borsa P, Chikhi L, Raufaste N, Bonhomme F. 2004. Genetix 4.05. Laboratoire Génome, Populations, Interactions, CNRS UMR 5000, Université de Montpellier II : Montpellier, France.

Benoit L, Born C, Vignes H, Chevallier MH, Todou G, Debain C, Joly Hélène I. 2011. Polymorphic microsatellite loci from Dacryodes edulis (Burseraceae), a Central Africa rainforest and fruit - tree species. American Journal of Botany, 2011: e74e75.

Garcia F, Noyer L, Risterucci AM, Chevallier MH. 2004. Genotyping of mature trees of Entandrophragma cylindricum with microsatellites. J. Hered., 95: 454-457.

Kengue J. 2002. Safou (Dacryodes edulis (GDon) HJ. Lam). International Centre for Underutilised Crops: Southampton, UK; 147 p.

Kengue J, Tchuenguem FFN, Adewusi HG. 2002. Towards the improvement of the African plum (Dacryodes edulis): Population variation and reproductive biology. Forest, Trees and Livelihoods, 12(1-2): 74-84.

Lam HJ. 1932. Beiträge zur morphologie der Burseraceae. Insbesondere der Canarieae. Ann. Jard. Bot. Buitenzorg, 42: 202 p.

Lovett P, Haq N. 2000. Diversity of Sheanut tree (Vitellaria paradoxa C.F Gaertn) in Ghana. Genetic Resources and Crop Evolution, 47: 293-304.

Mbofung CMF, Silou T, Mouaragadja I. 2002. Chemical characterisation of safou (Dacryodes edulis) and evaluation of its potential as an ingredient in nutritious biscuits. Forests, Trees and Livelihoods, 12: 105-117.

Okafor JC. 1983. Variety delimitation in Dacryodes edulis (G. Don) H. J. Lam (Burseraceae). Int. Trees Crop J., 2: 255265.

Onana JM. 1998. Les Burséracées du Cameroun : taxonomie, biométrie foliaire et biologie. Thèse de Doctorat de 3e cycle, Univ. Yaoundé, Cameroun, 267 p. 
Onana JM. 2008. A synoptic revision of Dacryodes (Burseraceae) in Africa, with a new species from Central Africa. Kew Bul., 63: 385-400.

Onana JM, Cheek M. 2003. A new species of Dacryodes (Burseraceae) from Zambia. Kew Bull., 58: 219 - 224.

Pierlot R. 1996. Deux nouvelles espèces de Dacryodes (Burseraceae) du Zaïre. Bull. Jard. Bot. Natl. Belg., 65: 359 - 367.

Schreckenberg K, Degrande A, Mbosso C, Boli Baboulé Z, Boyd C, Enyong L, Kanmegne J, Ngong C. 2002. The social and economic importance of Dacryodes edulis (G. Don) H.J. Lam in Southern Cameroon. Forests, Trees and Livelihoods, 12(1-2): 15-40.

Tabuna H. 1999. Le marché des produits forestiers non-ligneux de l'Afrique Centrale en France et en Belgique: produits, acteurs, circuits de distribution, débouchés actuels. CIFOR, 19: $32 \mathrm{p}$.

Tabuna H. 2000. Les produits forestiers non ligneux alimentaires de l'Afrique
Centrale sur les marchés français et belge. PhD. Thesis, Museum Nationan d'Histoire Naturelle de Paris.

Todou G., Doumenge C. 2008. Dacryodes buettneri (Engel) H.J. Lam. In Plant Resources of Tropical Africa (PROTA) : Timbers 7 (1). Louppe D, OtengAmoako AA, Brink M (eds), PROTA Fondation Backhuys Publishers, Leiden, Netherlands / CTA: Wageningen, Netherlands; $203-207$.

Verheij EWM. 2002. Dacryodes edulis (G.Don) H.J.Lam. In PROTA (Plant Resources of Tropical Africa / Ressources Végétales de l'Afrique Tropicale), Oyen LPA, Lemmens RHMJ (eds). Edeseweg 72 : Wageningen, Pays Bas.

Wright S. 1978. The interpretation of population structure by F-statistics with special regard to systems of mating. Evolution, 19: 395-420. 